\title{
Special issue on transit planning, operation and management in densely populated areas
}

\author{
Fumitaka Kurauchi · Jan-Dirk Schmöcker
}

Published online: 5 August 2010

(C) Springer Science+Business Media, LLC. 2010

Public transportation (PT) is the backbone of the private transportation in many metropolitan areas around the world. The reasons for this are well known and include equity, accessibility, efficiency, capacity as well as environmental ones. Nevertheless, in another, also large group of metropolitan areas public transportation has only a minor role or its existing role is declining. Several reasons for this are also well known and include operational inefficiency, service inflexibility, private traffic congestion reducing PT efficiency and several other reasons reducing service attractiveness for passengers such as crowding, safety and security, cleanliness etc. In general, in cities with higher population densities public transportation services can operate more efficiently by concentrating demand to fewer stops. Besides, often high density areas suffer from more traffic congestion and with it air pollution, therefore making high density cities the prime target for good PT.

Therefore, especially in several Asian areas where urban growth is among the world's largest and where often populations concentrate on a few central cities, there is ample opportunity for public transportation systems to be operated efficiently. Unfortunately, this is in many cases not happening, rather cities are moving towards increasing automobility. Figure 1 is taken from Van (2009) illustrating the strong common trend of several Asian cities towards increased car ownership with increasing GDP.

Van (2009) discusses that an important reason for this trend is that in many developing Asian countries the private car is seen as a status symbol. Therefore, despite heavy road congestion, one can expect this trend to continue, especially when disposable incomes are increasing in the next decades. In particular in many densely populated Asian cities one of the reason for a bad PT image is further crowding on stations and in vehicles. This increases people's willingness to shift to private transport and in particular to cars, if they can afford to do so. In order to avoid such a shift to car-oriented societies, it is hence

\footnotetext{
F. Kurauchi $(\bowtie)$

Department of Civil Engineering, Gifu University, 1-1, Yanagido, Gifu 501-1193, Japan

e-mail: kurauchi@gifu-u.ac.jp

J.-D. Schmöcker

Department of Urban Management, Kyoto University, C1-2-431, Katsura Nishikyo-ku,

Kyoto 615-8552, Japan

e-mail: schmoecker@trans.kuciv.kyoto-u.ac.jp
} 
Fig. 1 Car ownership and GDP per capita for Asian Pacific cities. Source: Van (2009)

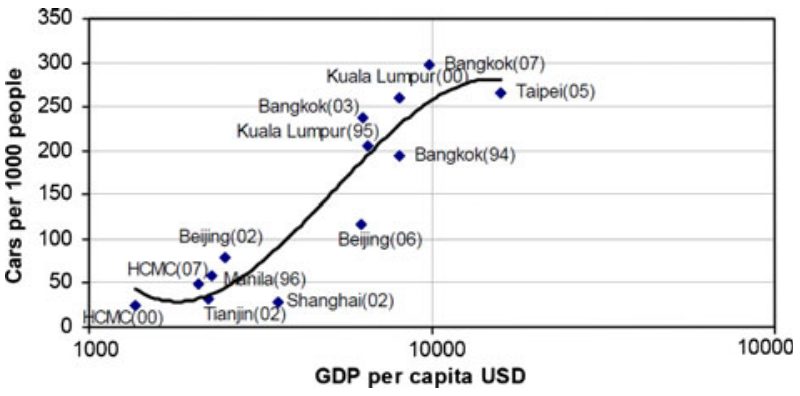

important to improve the image of the PT system, not least by spending often limited resources in an optimal way, so that the service is improved and capacity bottlenecks are removed.

This special issue therefore includes five papers that discuss strategies to improve public transportation operations: The first paper by Xu et al. reviews the history of PT in Beijing and aims to understand the importance of government influence on public transport uptake. The second paper by Kusakabe et al. shows how smart card data, which are now becoming increasingly available in many cities, can be used to analyse the routes taken by passengers. Such data can then be used to refine the timetables to improve service efficiency. Similarly, to fine-tune timetables to the passenger demand Li et al discuss that recent advances in activity based modelling could also have an influence on service frequency design. Asymmetric timetables could help to serve complex travel demands when telecommuting and flexible working times become the norm rather than the exception. Shimamoto et al. illustrate with a case study from Hiroshima City the effects of a trade between operator and passenger objectives on the optimal network design. Besides, accuracy also computational speed is a key to model the impact of scenarios to improve existing services. This is the motivation of the fourth paper by Kato et al. They compare different PT assignment models by applying them to the large Tokyo metropolitan area and provide some general conclusions on the advantages and drawbacks of the applied methods.

Clearly, these five papers only provide a snapshot of current research activities with the final aim to improve public transport in our cities. In summary, the five papers in this special issue touch on the effect of government influence, ITS developments, changing travel patterns, network design as well as available modelling tools. Other approaches to improve PT in large cities that are not discussed at all in this special issue include, for example, various forms of demand management, optimizing operational constraints or addressing intermodal issues and traffic congestion effects on road based PT. Further, "soft" approaches that are worth mentioning and sometimes require less capital investments are, among others, improving the PT service image, providing better service information as well as campaigns to raise awareness of environmental issues. As always, there is clearly scope for much further research.

\section{Reference}

Van, T.H.: A study on the applicability of mobility management to change the behavior of private mode use in Asian countries. PhD Thesis, Department of Civil Engineering, Tokyo Institute of Technology, Japan (2009) 


\section{Author Biographies}

Fumitaka Kurauchi is an Associate Professor in the Department of Civil Engineering at Gifu University. He received his Doctor of Engineering Degree from Kyoto University in 2002. His research interests include traffic control and operation on urban expressway networks, transport network reliability analysis and evaluation, and public transport network assignment. He is also interested in more flexible public transport services such as dial-a-ride using ICT technologies.

Jan-Dirk Schmöcker is an Associate Professor in the Department of Urban Management at Kyoto University, where he is teaching in a new "Global Engineering" degree program. Previously he was working at Tokyo Institute of Technology and at Imperial College London where he also obtained his PhD. His main research interests concern network reliability issues with a particular focus on public transport. In addition, triggered through projects with Transport for London, he has been looking into public transport uptake and mobility options of seniors. 